
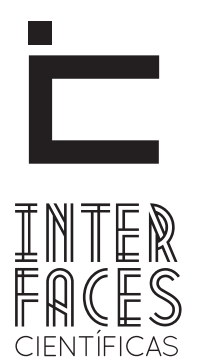

EXATAS E TECNOLÓGICAS

ISSN IMPRESSO - 2359-4934

ISSN ELETRÔNICO - 2359-4942

DOI - DOI - 10.17564/2359-4934.2015v1n3p9-22

\title{
USO DE AMBIENTES VIRTUAIS DE APRENDIZAGEM COMO SUPORTE AO ENSINO DE PROGRAMAÇÃO: UMA REVISÃO SISTEMÁTICA
}

André Santos Oliveira ${ }^{1}$

Adicinéia Aparecida de Oliveira ${ }^{3}$
Levi da Costa Mota ${ }^{2}$

\section{RESUMO}

Um dos grandes desafios enfrentados pelos cursos de computação é tornar o ensino de programação mais atrativo e intuitivo para os alunos. Diante disso, o uso de ambientes virtuais de aprendizagem (AVA) tem se colocado como uma solução para suportar e facilitar o ensino de programação. Este artigo descreve uma revisão sistemática da literatura cujo objetivo é identificar, analisar e avaliar estudos primários envolvendo uso de ambientes virtuais de aprendizagem como suporte ao ensino de programação com o propósito de caracterizá-las, do ponto de vista dos desenvolvedores e professores de programação no contexto no qual os estudos foram realizados. 0 trabalho constata que há apenas dois estudos relevantes publicados sobre o tema e que dessa forma há espaço para estudos futuros que busquem desenvolver novas técnicas e ferramentas pedagógicas que suportem com mais eficiência e eficácia os desafios enfrentados no ensino de programação.

\section{PALAVRAS-CHAVE}

Ensino. aprendizagem. linguagem de programação. AVA. ambiente virtual de aprendizagem. 


\section{ABSTRACT}

A major challenge faced by computer courses is to make programming education more attractive and intuitive for students. Thus, the use of virtual learning environments (VLE) has been placed as a solution to support and facilitate the teaching of programming. This article describes a systematic literature review that purpose is to identify, analyze and evaluate primary studies involving use of virtual learning environments as support for programming education in order to characterize them, from the point of view of program developers and teachers in context in which the studies were conducted. The paper notes that there are only two relevant published studies on the subject and that way there is room for future studies that seek to develop new techniques and educational tools that support more efficiently and effectively the challenges in teaching programming.

\section{KEYWORDS}

Education. Learning. Programming Language. VLE. Virtual Learning Environment.

\section{RESUMEN}

Un gran desafío que enfrentan los cursos de informática es hacer más atractiva e intuitiva la enseñanza de programación para los estudiantes. Por lo tanto, el uso de entornos virtuales de aprendizaje (EVA) se ha colocado como una solución para apoyar y facilitar la enseñanza de la programación. En este artículo se describe una revisión sistemática de la literatura cuyo objetivo es identificar, analizar y evaluar los estudios primarios que implican el uso de EVA como apoyo al estudio de programación con el fin de caracterizarlos, desde el punto de vista de los desarrolladores de programas y maestros en el contexto en el que se realizaron los estudios. El documento señala que sólo hay dos estudios relevantes publicados sobre el tema y de esa manera no hay espacio para futuros estudios que busquen desarrollar nuevas técnicas y herramientas educativas, apoyando de forma más eficiente y eficaz los retos en la enseñanza de programación.

\section{PALABRAS CLAVE}

enseñanza, aprendizaje, lenguaje de programación, EVA, entorno virtual de aprendizaje. 


\section{INTRODUÇÃ̃O}

Um dos principais problemas enfrentados pelos cursos de computação em geral são as dificuldades encontradas pelos alunos no aprendizado das abstrações das disciplinas de programação (MOHOROVIČIĆ; STRČIĆ, 2011). Isso é um dos fatores que podem conduzir a altas taxas de reprovação e a altos índices de evasão (CHAVES; CASTRO; LIMA; FERREIRA, 2014), devido à diminuição da autoestima do aluno e assim gerar o desinteresse pelos cursos.

Além disso, outros fatores influenciam a dificuldade no ensino de programação como: heterogeneidade do nível do conhecimento, por exemplo, uma fraca base matemática, dificuldades para a compreensão do problema e entendimento do assunto (CHAVES; CASTRO; LIMA; FERREIRA, 2014), expectativas e o ritmo de aprendizagem dos alunos, acompanhamento individual do aluno pelo professor, ambientes diversos de aprendizado não integrados, elaboração de atividades práticas e falta de respostas rápidas às práticas dos alunos (CHAVES; CASTRO; LIMA; FERREIRA, 2013, 2014).

Então, a fim de produzir melhores resultados em processos de aprendizagem nas disciplinas de programação, há necessidade de modificar os métodos atuais de ensino, que permitam tornar o processo de ensino-aprendizagem mais atrativo para o aluno (LAW; LEE; YU, 2010).

Existem vários tipos de abordagens para o ensino de programação, mas o uso de Ambientes Virtuais de Aprendizagem (AVA) surge como uma proposta para ajudar a resolver estas dificuldades, para, por meio de suas funcionalidades e adaptações fornecer suporte ao ensino de programação (CHAVES; CASTRO; LIMA; FERREIRA, 2013, 2014).

Entretanto, dentro desse contexto, o presente artigo apresenta uma revisão sistemática com o intuito de identificar, analisar e avaliar estudos primários publicados, envolvendo uso de ambientes virtuais de aprendizagem como suporte ao ensino de programação, visando identificar o estado da arte e de contri- buir com futuros estudos e trabalhos acadêmicos que busquem o uso dessa abordagem para alcançar melhores resultados para o ensino de programação.

0 artigo está organizado em seis seções. Na seção 2 é feita uma revisão bibliográfica onde são analisados alguns estudos para fundamentar os conceitos sobre os assuntos abordados neste estudo, é apresentada uma visão geral sobre o ensino de programação e seus problemas, bem como o uso da abordagem de Ambientes Virtual de Aprendizagem para tentar resolvê-los. Na seção 3, é descrito o protocolo que foi utilizado na revisão sistemática. Na seção 4, são apresentados os resultados da revisão sistemática. Na seção 5, é realizada a análise dos dados, assim como discussão dos resultados obtidos na revisão sistemática.

Por fim, a seção 6 traz as conclusões do trabalho.

\section{REVISÃO BIBLIOGRÁFICA}

Nesta seção será feita uma revisão bibliográfica com o objetivo de levantar e entender melhor alguns dos conceitos e estudos relevantes sobre o assunto a fim de contribuir para fundamentar a revisão sistemática da literatura, fruto deste artigo.

\subsection{ENSINO DE PROGRAMACÃO}

Programação exige o uso de complexas habilidades cognitivas, como raciocínio, resolução de problema, planejamento e criatividade, isto é, desenvolver representações de um problema sob a forma de estruturas lógicas, que são traduzidas em códigos, utilizando-se de uma linguagem formal (MOHOROVIČIĆ; STRČIĆ, 2011).

Mohorovičić; Strčić (2011) e diversos autores concordam que o ensino de programação representa um desafio para vários estudantes, pois eles se deparam com muitos conceitos de difícil compreensão. Isso é um dos fatores que podem conduzir a altas taxas de reprovação e a altos índices de evasão, devido à diminuição da autoestima e consequente geração de desinteresse por parte dos alunos (CHAVES; CASTRO; LIMA; FERREIRA, 2014). 
Há diversos fatores que influenciam e dificultam o ensino de programação, como:

- Heterogeneidade do nível do conhecimento dos alunos: há alunos com os mais diversos tipos de conhecimentos que não estão nivelados. Alguns desses conhecimentos são pré-requisitos para o ensino de programação e não são de domínio do aluno. Uma fraca base matemática e dificuldades para a compreensão de problemas são frequentemente encontrados pelos professores durante o processo de ensino-aprendizagem de programação (CHAVES; CASTRO; LIMA; FERREIRA, 2014);

- Expectativas e o ritmo de aprendizagem dos alunos: devido heterogeneidade do nível do conhecimento dos alunos (explicado no tópico anterior), cada aluno tem uma ou várias expectativas e um ritmo de aprendizado que devem ser observados e considerados pelo professor (LAW; LEE; YU, 2010);

- Acompanhamento individual do aluno pelo professor: em turmas com vários alunos é difícil conseguir individualizar o aprendizado para o aluno. Os autores De Barros Paes; Malaquias; Guimarães; Almeida (2013) e França; Soares (2011) abordam essa preocupação em seus trabalhos;

- Ambientes diversos de aprendizado não integrados: algumas abordagens de ensino-aprendizagem buscam utilizar ambientes de aprendizado que minimizem alguns desses problemas relatados, mas na maioria das vezes confundem os alunos, pois eles precisam acessar vários ambientes, não interligados, para conseguir compreender o conteúdo (CHAVES; CASTRO; LIMA; FERREIRA, 2013, 2014);

- Elaboração de atividades práticas: elaborar atividades práticas exige tempo do professor que na maioria das vezes está envolvido com várias turmas e com outras atividades pedagógicas, então esse é um problema que o professor deve gerenciar (CHAVES; CASTRO; LIMA; FERREIRA, 2013);

- Falta de respostas rápidas às práticas (DE BARROS PAES; MALAQUIAS; GUIMARÃES; ALMEIDA, 2013) e dúvidas dos alunos: uma das dificuldades dos professores é possuir ferramentas que ajudem a facilitar as práticas de ensino de programação extraclasse e que consigam dirimir as dúvidas no momento da tentativa do aprendizado (CHAVES; CASTRO; LIMA; FERREIRA, 2014).

Preocupar-se apenas com transmissão de conhecimento não é suficiente para quem quer ensinar programação (LAW; LEE; YU, 2010). Os estudos relatam que é importante identificar um estilo correto de ensino-aprendizagem de programação, procurar por abordagens de Tecnologia da Informação (TI) que aproximem o aluno e professor.

Dessa forma, devem-se procurar formas de motivação, são fatores que podem permitir que um aluno adquira as habilidades de programação com rapidez e mais facilidade, gerando assim o interesse dele pelo conteúdo (LAW; LEE; YU, 2010).

Uma das abordagens para tentar melhorar e atrair o aluno no ensino de programação se dá por meio do uso de Ambientes Virtuais de Aprendizagem como suporte ao ensino de programação que será abordado no próximo tópico.

\subsection{AMBIENTE VIRTUAL DE APRENDIZAGEM}

A evolução dos recursos tecnológicos e de comunicação na área de Tecnologia da Informação (como a popularização da Internet) está mudando a forma como o ensino-aprendizagem deve ser abordado para atender as novas demandas de aprendizagem, dessas pessoas envolvidas nesse novo mundo moderno (PEREIRA; SCHMITT; DIAS, 2007).

Diante desse cenário, os Ambientes Virtuais de Aprendizagem (AVA) surgem como uma proposta para ajudar a resolver estas dificuldades (PEREIRA; SCHMITT; DIAS, 2007).

AVA consiste em um conjunto de ferramentas e recursos tecnológicos integrados que permitem mediar o processo ensino-aprendizagem seja ele presencial ou à distância; permite, ainda, gerenciar e administrar os variados aspectos da aprendizagem, como disponibilizar conteúdos, cursos, acompanhar o aluno (por meio de fóruns, mensagens, e-mail, chat, whiteboard), avaliar o processo de ensino-aprendizagem e promover a produção de atividades individuais ou em grupo (PEREIRA; SCHMITT; DIAS, 2007). 
Aproveitando-se do conjunto de ferramentas e recursos tecnológicos integrados ao AVA, há algumas iniciativas que propõem integrar ferramentas necessárias para suportar o ensino de programação (CHAVES; CASTRO; LIMA; FERREIRA, 2014), como:

- Disponibilização de editores e compiladores de algumas linguagens de programação (FRANÇA; SOARES, 2011 e DE BARROS PAES; MALAQUIAS; GUIMARÃES; ALMEIDA, 2013);

- Utilização de base de dados de questões disponíveis em juízes online. Juízes online que são repositórios de problemas de programação, utilizadas em maratonas de programação, além de possibilitar a submissão dessas questões para avaliações on-line, retornando assim o resultado do código enviado (VERDÚ; REGUERAS; VERDÚ; LEAL; DE CASTRO; QUEIRÓS, 2012 e CHAVES; CASTRO; LIMA; FERREIRA, 2013, 2014);

- Automatização dos processos de submissão e avaliação das atividades práticas dos alunos. Por meio, do uso de juízes on-line, explicado no tópico anterior, onde eles podem ser acessados para submeter as respostas elaboradas pelos alunos para avaliação dos juízes e eles retornam o resultado do código enviado (CHAVES; CASTRO; LIMA; FERREIRA, 2013, 2014);

- Gerenciar e organizar os problemas de programação por assunto, grau de dificuldade, permitindo a votação do nível de dificuldade dos problemas pelos alunos ou por meio da análise de quantidade de respostas submetidas (DE BARROS PAES; MALAQUIAS; GUIMARÃES; ALMEIDA, 2013).

Essas ferramentas integradas permitem que 0 aluno tenha um ambiente único de aprendizagem que suportem atividades de programação com todas as ferramentas pedagógicas integradas e em um só lugar (VERDÚ; REGUERAS; VERDÚ; LEAL; DE CASTRO; QUEIRÓS, 2012 e CHAVES; CASTRO; LIMA; FERREIRA, 2013, 2014).

Essa integração possibilita resolver os principais problemas envolvidos nas atuais abordagens de ensino, onde o aluno poderá: ditar o seu ritmo de aprendizado, ter o seu acompanhamento individualizado pelo professor, possibilitar a interação com outros alunos e onde o mesmo pode ter as respostas às dúvidas e práticas de programação mais rapidamente. Para o professor, permite acompanhar as atividades do aluno de forma mais individualizada e de gerenciar as atividades de ensino-aprendizagem (VERDÚ; REGUERAS; VERDÚ; LEAL; DE CASTRO; QUEIRÓS, 2012 e CHAVES; CASTRO; LIMA; FERREIRA, 2013, 2014).

Atualmente, existem algumas iniciativas de pesquisa que procuram fazer uso de Ambientes Virtuais de Aprendizagem para suportar o ensino à programação.

A Tabela 1 mostra um resumo sumarizado desses ambientes encontrados nesse estudo, bem como algumas informações relevantes sobre elas.

\section{PROTOCOLO DA REVISÃO SISTEMÁTICA}

Esta seção contém o protocolo baseado em Mafra (2005) e Kitchenham (2002), o qual foi utilizado para aplicação da revisão sistemática, cujo foco das buscas concentrou-se na identificação das pesquisas que apresentam ambientes virtuais de aprendizagem que servissem de suporte ao ensino de programação.

\subsection{FORMULACÃO DAS PERGUNTAS}

Está seção apresenta as perguntas utilizadas para a busca dos trabalhos relacionados.

Questão 1. Quais são os ambientes virtuais de aprendizagem que são utilizados como suporte ao ensino de programação?

Questão 2. Onde esses ambientes educacionais integrados são utilizados: em que país, em que instituição de ensino?

Questão 3. Quais são as linguagens de programação utilizadas nesses ambientes, para o aprendizado no ensino de programação?

Questão 4. Esse ambiente possui ou está integrado a algum serviço de juízes on-line? 


\subsection{CRITÉRIOS DE SELEÇ̃̃O DE FONTES}

- Ferramentas de busca por meio de palavras-chaves;

- Consulta das publicações disponíveis através da WEB.

\subsection{CRITÉRIOS DE INCLUSÃO}

Os critérios de inclusão foram:

- Artigos publicados nos últimos 10 anos (entre 2005 e 2015);

- As publicações devem apresentar textos completos e disponíveis para download;
- Deveriam apresentar trabalhos sobre o uso de ambientes virtuais de aprendizagem ou ambientes de aprendizado WEB como suporte para o ensino de programação;

- Deveriam estar escritos em inglês ou português;

- Análise dos pesquisadores sobre a relevância das publicações para o estudo em questão.

\subsection{CRITÉRIOS DE EXCLUSÃO}

Os critérios de exclusão foram:

- Garantia de resultados únicos, ou seja, não utilizar publicações duplicadas;

Tabela 1 - Estudos sobre AVAs como suporte ao Ensino de Programação

\begin{tabular}{|c|c|c|c|c|c|c|c|c|}
\hline \multirow[t]{2}{*}{ Solução } & \multirow[t]{2}{*}{ AVA } & \multirow{2}{*}{$\begin{array}{l}\text { Onde } \\
\text { está } \\
\text { sendo } \\
\text { usado? }\end{array}$} & \multicolumn{5}{|c|}{ Recursos para Ensino de Programação } & \multirow{2}{*}{$\begin{array}{c}\text { Artigos } \\
\text { Relacionados }\end{array}$} \\
\hline & & & $\begin{array}{c}\text { Casos } \\
\text { de Teste } \\
\text { Automático }\end{array}$ & $\begin{array}{l}\text { Juízes } \\
\text { Online }\end{array}$ & $\begin{array}{l}\text { Linguagem } \\
\text { suportada }\end{array}$ & $\begin{array}{l}\text { Defini- } \\
\text { ção de } \\
\text { Nivel de } \\
\text { Dificul- } \\
\text { dade da } \\
\text { Questão }\end{array}$ & Outros & \\
\hline TheHuxley & $N / I$ * & $\begin{array}{l}\text { Univer- } \\
\text { sidade } \\
\text { Fede- } \\
\text { ral de } \\
\text { Ala- } \\
\text { goas - } \\
\text { Brasil }\end{array}$ & $N / l$ & $\begin{array}{l}\text { Possui } \\
\text { ava- } \\
\text { liação } \\
\text { auto- } \\
\text { mática } \\
\text { nativa }\end{array}$ & $\mathrm{N} / \mathrm{I}$ & Possui & $\begin{array}{l}\text { Detec- } \\
\text { ção de } \\
\text { plágio; } \\
\text { Uso de } \\
\text { jogos }\end{array}$ & $\begin{array}{l}\text { (DE BAR- } \\
\text { ROS PAES; } \\
\text { MALAQUIAS; } \\
\text { GUIMARÃES; } \\
\text { ALMEIDA, } \\
\text { 2013) }\end{array}$ \\
\hline PASS & $\mathrm{N} / \mathrm{I}$ & $\begin{array}{l}\text { Univer- } \\
\text { sidade } \\
\text { de } \\
\text { Hong } \\
\text { Kong - } \\
\text { China }\end{array}$ & $N / \mathrm{I}$ & $\begin{array}{l}\text { Possui } \\
\text { ava- } \\
\text { liação } \\
\text { auto- } \\
\text { mática } \\
\text { nativa }\end{array}$ & $\mathrm{N} / \mathrm{I}$ & $\mathrm{N} / \mathrm{I}$ & & $\begin{array}{l}\text { (LAW; LEE; } \\
\text { YU, 2010) }\end{array}$ \\
\hline EduJudge & Moodle & N/I & Possui & $\begin{array}{l}\text { Inte- } \\
\text { gra-se } \\
\text { a UVA } \\
\text { Online } \\
\text { Judge }\end{array}$ & $\begin{array}{l}\text { Pascal, } \\
\text { Java, C e } \\
\text { C++ }\end{array}$ & $\mathrm{N} / \mathrm{I}$ & $\begin{array}{l}\text { Integra } \\
\text { com } \\
\text { ferra- } \\
\text { menta } \\
\text { de } \\
\text { apren- } \\
\text { dizado } \\
\text { com- } \\
\text { petitivo }\end{array}$ & $\begin{array}{l}\text { (VERDÚ; } \\
\text { REGUERAS; } \\
\text { VERDÚ; } \\
\text { LEAL; DE } \\
\text { CASTRO; } \\
\text { QUEIRÓS, } \\
\text { 2012) }\end{array}$ \\
\hline
\end{tabular}




\begin{tabular}{|c|c|c|c|c|c|c|c|}
\hline BOCA-LAB & Moodle & $\begin{array}{l}\text { Univer- } \\
\text { sidade } \\
\text { Fede- } \\
\text { ral do } \\
\text { Ceará } \\
\text { - Bra- } \\
\text { sil }\end{array}$ & $\mathrm{N} / \mathrm{I}$ & $\begin{array}{l}\text { Inte- } \\
\text { gra-se } \\
\text { ao } \\
\text { BOCA }\end{array}$ & $\begin{array}{l}\text { Java, C e } \\
\mathrm{C}++\end{array}$ & $N / I$ & $\begin{array}{l}\text { (CHAVES; } \\
\text { CASTRO; } \\
\text { LIMA; FER- } \\
\text { REIRA, 2014) } \\
\text { (FRANÇA; } \\
\text { SOARES, } \\
\text { 2011) } \\
\text { (CHAVES; } \\
\text { CASTRO; } \\
\text { LIMA; LIMA; } \\
\text { FERREIRA; } \\
\text { 2013) }\end{array}$ \\
\hline MOJO & Moodle & $N / I$ & $\mathrm{~N} / \mathrm{I}$ & $\begin{array}{l}\text { Inte- } \\
\text { gra-se } \\
\text { ao } \\
\text { SPOJ } \\
\text { Brasil e } \\
\text { ao URI } \\
\text { Online } \\
\text { Judge }\end{array}$ & $N / I$ & $\mathrm{~N} / \mathrm{I}$ & $\begin{array}{l}\text { (CHAVES; } \\
\text { CASTRO; } \\
\text { LIMA; FER- } \\
\text { REIRA, 2014) } \\
\text { (CHAVES; } \\
\text { CASTRO; } \\
\text { LIMA; LIMA; } \\
\text { FERREIRA, } \\
\text { 2013) }\end{array}$ \\
\hline ProgTest & Moodle & $\begin{array}{l}\text { Univer- } \\
\text { sidade } \\
\text { de São } \\
\text { Paulo - } \\
\text { Brasil }\end{array}$ & Possui & $\begin{array}{l}\text { Possui } \\
\text { ava- } \\
\text { liação } \\
\text { auto- } \\
\text { mática } \\
\text { nativa }\end{array}$ & Java e C & $\mathrm{N} / \mathrm{I}$ & $\begin{array}{l}\text { (CHAVES; } \\
\text { CASTRO; } \\
\text { LIMA; FER- } \\
\text { REIRA, 2014) } \\
\text { (CHAVES; } \\
\text { CASTRO; } \\
\text { LIMA; LIMA; } \\
\text { FERREIRA, } \\
\text { 2013) }\end{array}$ \\
\hline OCLS & $\mathrm{N} / \mathrm{I}$ & & $N / I$ & $N / I$ & Java e C++ & $N / I$ & $\begin{array}{l}\text { (OTHMAN; } \\
\text { OTHMAN, } \\
2012 \text { ) }\end{array}$ \\
\hline
\end{tabular}

(*) Não Identificado. 
Não sejam trabalhos de conclusão de curso ou dissertações;

Ambiente virtual de aprendizagem ou ambientes de aprendizado WEB apenas citados nas publicações não fazem parte deste estudo.

\subsection{BUSCA}

Foram consultadas as bases a seguir: Periódicos da CAPES (IEEE journals, IEEE conferences, ACM journals, ACM conferences, Kluwer journals e Elsevier journals); IEEExplorer.

Para as perguntas relacionadas nesta pesquisa, as Strings de buscas foram:

String 1: ava $<O R>$ "ambiente virtual de aprendizagem" $<A N D>$ ensino $<O R>$ aprendizagem $<O R>$ curso <AND> “linguagem de programação" <OR > programação;

String 2: vle $\langle\mathrm{OR}\rangle$ virtual learning environment $<A N D>$ learn $<O R>$ teaching $<O R>$ learning $<O R>$ course <AND> programming language $<\mathrm{OR}>$ programming;

String 3: learning environment $<O R>$ learning environments $<\mathrm{OR}>$ "vle" $<\mathrm{OR}>$ virtual learning environment $\langle O R>$ virtual learning environments <AND> "learning programming $\angle O R>$ educational programming $<\mathrm{OR}>$ course programming.

\section{RESULTADOS DA REVISÃO SISTEMÁTICA}

Após a aplicação dos métodos de seleção dos trabalhos, definidos previamente no protocolo de revisão sistemática, foram obtidas duas publicações, como resultado da seleção, como mostra a Tabela 2. Nessa tabela, a coluna "String de busca" exibe as Strings de busca automática usadas nas pesquisas das publicações. As colunas de cabeçaIho, CAPES e IEEE, exibem as quantidades da busca (antes e depois dos critérios de seleção) em cada motor de pesquisa, e a coluna intitulada TOTAL, apresenta a soma da quantidade dos trabalhos encontrados em todo o processo de pesquisa.
Foram, também, elaborados gráficos que mostram os resultados da execução das pesquisas realizadas. A Figura 1, mostra a quantidade total de publicações encontradas pelas Strings, demonstrando a eficácia de cada uma delas, e a quantidade de publicações selecionadas após a aplicação dos critérios de seleção.

Ainda pode-se observar na Figura 1, que a consulta com as palavras chaves em português trouxe uma quantidade menor de resultados do que a consulta utilizando as outras Strings que utilizam palavras chaves em inglês. Dessa forma, supõe-se que há menos trabalhos publicados na língua portuguesa do que trabalhos em inglês.

Figura 1 - Publicações por String de busca

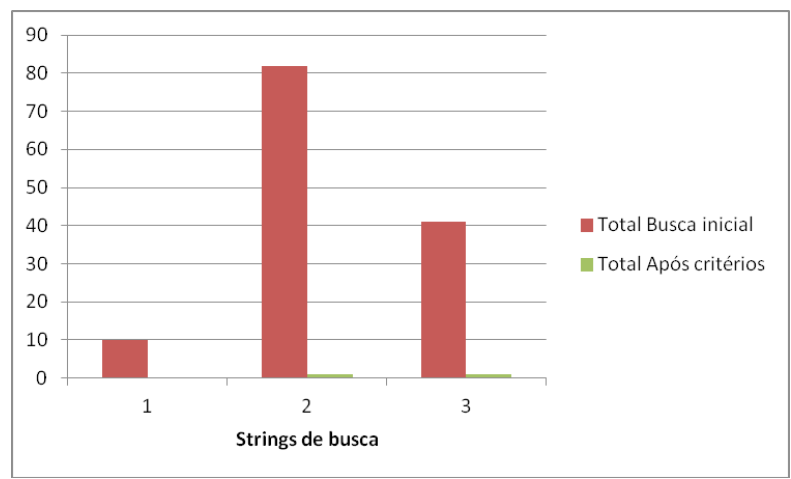

A Figura 2 mostra a quantidade total de publicações encontradas por motor de busca automática, demonstrando a eficácia de cada uma delas, e a quantidade de publicações selecionadas após a aplicação dos critérios de seleção, inclusão e exclusão.

Ainda, foi elaborado um gráfico entre o período de 2005 a 2015, exibido na Figura 3, a qual mostra as publicações encontradas de acordo com o ano de publicação dos mesmos. Percebeu-se, dentro do período de pesquisa, que apenas no ano de 2012 foram encontrados estudos relevantes e que atenderam aos critérios de seleção, inclusão e exclusão definidos. 
Tabela 2 - Resultados das quantidades de publicações selecionadas pelas buscas do protocolo

\begin{tabular}{|c|c|c|c|c|c|c|c|}
\hline & String de busca & CAPES & & IEEE & & Total & \\
\hline & & $\begin{array}{l}\text { Busca } \\
\text { inicial }\end{array}$ & $\begin{array}{l}\text { Após } \\
\text { critérios } \\
\text { de seleção, } \\
\text { inclusão e } \\
\text { exclusão }\end{array}$ & $\begin{array}{l}\text { Busca } \\
\text { inicial }\end{array}$ & $\begin{array}{l}\text { Após cri- } \\
\text { térios de } \\
\text { seleção, } \\
\text { inclusão e } \\
\text { exclusão }\end{array}$ & $\begin{array}{l}\text { Busca } \\
\text { inicial }\end{array}$ & $\begin{array}{l}\text { Após } \\
\text { crité- } \\
\text { rios de } \\
\text { seleção, } \\
\text { inclusão } \\
\text { e exclu- } \\
\text { são }\end{array}$ \\
\hline 1 & $\begin{array}{l}\text { (ava OR “ambiente virtual de aprendi- } \\
\text { zagem”) AND (ensino OR aprendiza- } \\
\text { gem OR curso) AND (“linguagem de } \\
\text { programação” OR programação) }\end{array}$ & 10 & 0 & 0 & 0 & 10 & 0 \\
\hline 2 & $\begin{array}{l}\text { (vle OR “virtual learning environ- } \\
\text { ment”) AND (learn OR teaching OR } \\
\text { learning OR course) AND (“program- } \\
\text { ming language” OR programming) }\end{array}$ & 23 & 1 & 59 & 0 & 82 & 1 \\
\hline 3 & $\begin{array}{l}\text { (“learning environment” OR “learning } \\
\text { environments" OR “vle” OR “virtual } \\
\text { learning environment” OR “virtual } \\
\text { learning environments") AND (“lear- } \\
\text { ning programming" OR “educational } \\
\text { programming" OR "course program- } \\
\text { ming") }\end{array}$ & 27 & 1 & 14 & 0 & 41 & 1 \\
\hline & Total & 60 & 2 & 73 & 0 & 133 & 2 \\
\hline
\end{tabular}


Figura 2 - Publicações por base de busca

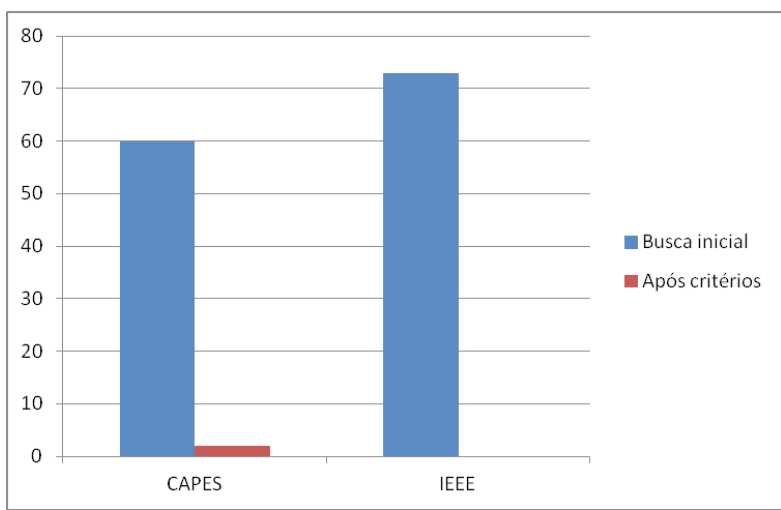

Figura 3 - Publicações após os critérios de seleção, inclusão e exclusão por ano

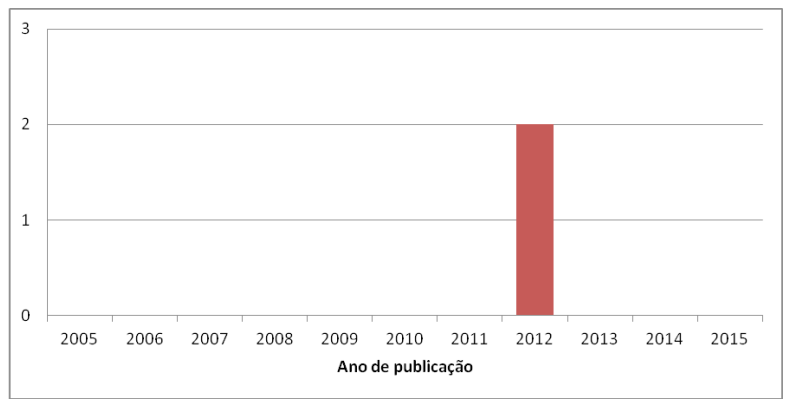

Dessa forma, pode-se perceber que faltam publicações científicas que abordem soluções de AVA para suportar o ensino de programação diante das bases pesquisadas e dos critérios de seleção, inclusão e exclusão estabelecidos.

\section{ANÁLISE DOS DADOS E DISCUSSÃO}

Após a execução do protocolo, foram identificados, inicialmente, 133 publicações, a partir das strings de busca utilizadas nas duas bases de dados consultadas. Aplicados os critérios de inclusão e exclusão na leitura das publicações, chegou-se ao número de duas publicações que foram selecionadas e analisa- das com o intuito de responder às quatro perguntas propostas na RS. Abaixo são mostrados os trabalhos selecionados e em seguida é apresentada uma visão geral de cada trabalho.

\subsection{RESUMO DOS TRABALHOS SELECIONADOS}

Foram selecionados os trabalhos de Othman; Othman (2012) e Verdú; Regueras; Verdú; Leal; De Castro; Queirós (2012).

O trabalho de Othman; Othman (2012) discute a proposta de um sistema de aprendizado virtual colaborativo, baseado numa solução que implementa um AVA para cursos de introdução à programação de computadores com base numa das técnicas chamada Think-Pair-Share. Essa técnica de aprendizagem colaborativa é baseada em três etapas: a primeira etapa chamada de "Think", cada um dos estudantes pensa em silêncio sobre uma questão dentro de um determinado período de tempo. Finalizado esse tempo, ocorre a fase de "Pair", onde os estudantes irão emparelhar-se e discutir, juntos, as suas possíveis respostas.

Depois, durante a fase final, que é a fase Share, os pares vão compartilhar suas respostas com os outros pares. 0 artigo, então, aborda o projeto de um modelo de aprendizagem colaborativa que faz uso de AVA e acrescenta recursos de comunicação entre os pares. Ele concentra nas etapas de construção do modelo, incluindo o levantamento dos requisitos com base em pesquisas realizadas com estudantes de programação e o que eles desejam numa solução desse tipo. Ao final, ele demonstra a eficácia do modelo colaborativo proposto no desempenho dos alunos que foram submetidos ao mesmo.

O trabalho de Verdú; Regueras; Verdú; Leal; De Castro; Queirós (2012) apresenta o EduJudge, um projeto com objetivo de integrar o UVA Online Judge, uma ferramenta de treino on-line para programação, ao Moodle, plataforma eficaz e sólida de Ambiente Virtual de Aprendizado, e ainda integrando com o QUESTOURnament, ferramenta de aprendizado baseado em competição. 
0 resultado é apresentado como um sistema capaz de permitir a educadores aplicarem diferentes abordagens pedagógicas para o ensino de linguagens, facilitando a busca de problemas, e provendo um mecanismo automático de submissão destes. 0 estudo, também, aponta novas estratégias de ensino para motivar alunos nesse tipo de aprendizado. São feitas análises do nível de motivação dos alunos após o uso da solução proposta, com resultados que apontam que tanto alunos quanto professores perceberam diversos benefícios trazidos no processo de aprendizagem.

\subsection{DISCUSSÕES SOBRE A PESQUISA}

Nesta seção serão respondidas as questões de pesquisa baseadas nos artigos selecionados.

\subsubsection{QUAIS SÃO OS AMBIENTES VIRTUAIS DE APRENDIZAGEM QUE SÃO UTILIZADOS COMO SUPORTE AO ENSINO DE PROGRAMAÇÃO?}

No estudo de Othman; Othman (2012), foi identificada a solução Online Collaborative Learning System (OCLS) que é o próprio AVA. No trabalho de Verdú; Regueras; Verdú; Leal; De Castro; Queirós (2012) foi apresentada a solução EduJudge que utiliza como AVA o Moodle e faz integração com outras ferramentas para suportar o ensino de programação.

\subsubsection{ONDE ESSES AMBIENTES EDUCACIONAIS INTEGRADOS SÃO} UTILIZADOS: EM QUE PAÍS, EM QUE INSTITUIÇÃO DE ENSINO?

O trabalho de Othman; Othman (2012), apresenta solução que foi usada nos centros acadêmicos de Universiti Teknologi MARA Perlis - Malásia e Universiti Tun Hussein Onn - Malásia. No trabalho de Verdú; Regueras; Verdú; Leal; De Castro; Queirós (2012) não foi identificado onde a solução está sendo utilizada.

\subsubsection{QUAIS SÃO AS LINGUAGENS DE PROGRAMAÇÃO UTILIZADAS NESSES AMBIENTES, PARA O APRENDIZADO NO ENSINO DE PROGRAMAÇ̃̃O?}

A solução que trata o trabalho de Othman; Othman (2012), dá suporte às linguagens de programação C++ e Java. Já o EduJudge, abordado por Verdú; Regueras; Verdú; Leal; De Castro; Queirós (2012) atende às linguagens Pascal, Java, C e C++.

\subsubsection{ESSE AMBIENTE POSSUI OU ESTÁ INTEGRADO A ALGUM SERVIÇO DE JUIZZES ON-LINE?}

No estudo de Othman; Othman (2012), não foi identificada nenhuma proposta ou integração com serviço de juízes on-line. No trabalho de Verdú; Regueras; Verdú; Leal; De Castro; Queirós (2012) a solução integra-se com o UVA On-line Judge para prover serviço de juízes online.

Na Tabela 3 são sumarizados os resultados da pesquisa abordados pela revisão sistemática que respondem às questões levantadas no protocolo. 
Tabela 3 - Resultado sumarizado das questões de pesquisa

\begin{tabular}{|c|c|c|c|c|c|}
\hline \multirow[b]{3}{*}{ Publicações } & \multirow[b]{3}{*}{ Solução } & \multicolumn{4}{|c|}{ Questões de pesquisa } \\
\hline & & Q1 & Q2 & Q3 & Q4 \\
\hline & & AVA & $\begin{array}{l}\text { Instituição } \\
\text { de ensino e } \\
\text { país }\end{array}$ & $\begin{array}{l}\text { Lingua- } \\
\text { gens de pro- } \\
\text { gramacão }\end{array}$ & $\begin{array}{l}\text { Juízes } \\
\text { Online }\end{array}$ \\
\hline $\begin{array}{l}\text { (OTHMAN; OTH- } \\
\text { MAN, 2012) }\end{array}$ & $\begin{array}{l}\quad \text { OCLS - On- } \\
\text { line Collabo- } \\
\text { rative Lear- } \\
\text { ning System }\end{array}$ & OCLS & $\begin{array}{l}\quad \text { Universiti } \\
\text { Teknologi } \\
\text { MARA Per- } \\
\text { lis - Malásia, } \\
\text { Universiti Tun } \\
\text { Hussein Onn - } \\
\text { Malásia }\end{array}$ & Java e C++ & $N / I^{*}$ \\
\hline $\begin{array}{l}\text { (VERDÚ; REGUE- } \\
\text { RAS; VERDÚ; LEAL; } \\
\text { DE CASTRO; QUEI- } \\
\text { RÓS, 2012) }\end{array}$ & EduJudge & Moodle & $\mathrm{N} / \mathrm{I}$ & $\begin{array}{c}\text { Pascal, } \\
\text { Java, C, C++ }\end{array}$ & $\begin{array}{l}\text { UVA Onli- } \\
\text { ne Judge }\end{array}$ \\
\hline
\end{tabular}

\section{CONCLUSÃO}

Este artigo apresentou uma Revisão Sistemática de Literatura, na qual foram buscadas e avaliadas, pesquisas acerca do uso de Ambientes Virtuais de Aprendizagem (AVA) como suporte ao ensino de programação, no sentido de conhecer estudos que as identificassem e caracterizassem.

Foi percebido que há poucos trabalhos publicados que tratam do objeto de pesquisa, conforme Tabela 1, que apresenta uma visão geral dos trabalhos encontrados e selecionados, com a identificação de apenas duas publicações.

Embora este trabalho tenha tentado identificar soluções que usem AVA como ambiente de suporte ao ensino de programação, não foi possível concluir quais são as principais ferramentas utilizadas.

Como trabalhos futuros, podem ser desenvolvidos estudos que busquem implementar novas técnicas e ferramentas pedagógicas que sejam mais intuitivas e interessante para os alunos e suportem com mais eficiência e eficácia os desafios enfrentados no ensino de programação.

\section{REFERÊNCIAS}

CHAVES, José Osvaldo; CASTRO Angélica; LIMA Rommel; LIMA Marcos Vinicius; FERREIRA, Karl. MOJO: Uma ferramenta para auxiliar o professor em disciplinas de programação. X Congresso Brasileiro de Ensino Superior a Distância (ESUD 2013), 2013.

CHAVES, José Osvaldo; CASTRO Angélica; LIMA Rommel; LIMA Marcos Vinicius; FERREIRA, Karl. Um Módulo de Integração com Juízes On-line para Auxiliar Atividades de Programação. Revista de Informática Aplicada, v.9, n.1, 2014.

DE BARROS PAES, Rodrigo; MALAQUIAS, Romero; GUIMARÃES, Márcio; ALMEIDA Hyggo. Ferramenta para a Avaliação de Aprendizado de Alunos em Programação de Computadores. Anais dos Workshops do Congresso Brasileiro de Informática na Educação, 2013.

FRANÇA, Allyson Bonetti; SOARES, José Marques. Sistema de apoio a atividades de laboratório de pro- 
gramação via Moodle com suporte ao balanceamento de carga. Anais do Simpósio Brasileiro de Informática na Educação (SBIE), Aracaju, 2011.

KITCHENHAM, et al, Preliminary Guidelines for Empirical Research in Software Engineering, IEEE Transactions on Software Engineering, v.28, n8, August 2002.

LAW, Kris MY; LEE, Victor CS; YU, Yuen-Tak. Learning motivation in e-learning facilitated computer programming courses. Computers \& Education, v.55, n.1, 2010. p.218-228.

\section{MAFRA, S. N. Protocolo de Revisão Sistemática:}

Grupo de Engenharia de Software Experimental, Programa de Engenharia de Sistemas e Computação (COPPE/UFRJ), 2005.
MOHOROVIČIĆ, Sanja; STRČIĆ, Vedran. An Overview of Computer Programming Teaching Methods. XXII

Central European Conference on Information and Intelligent Systems, 2011. p.1-6.

OTHMAN, Mahfudzah; OTHMAN, Muhaini. The Proposed Model of Collaborative Virtual Learning Environment for Introductory Programming Course. Turkish Online Journal of Distance Education-TOJDE, 13 (January), 2012. p.100-112.

PEREIRA, Alice Theresinha Cybis; SCHMITT, Valdenise; DIAS, M. R. A. C. Ambientes virtuais de aprendizagem. AVA-Ambientes Virtuais de Aprendizagem em Diferentes Contextos. Rio de Janeiro: Ciência Moderna, 2007.

VERDÚ, Elena; REGUERAS Luisa M.; VERDÚ Mária J.; LEAL José P.; DE CASTRO Juan P.; QUEIRÓS Ricardo. A distributed system for learning programming on-line. Computers \& Education, v.58, n.1, 2012. p. 1-10. 
